\section{Neurobiologie et mutagenèse ciblée in vivo}

\section{Les processus}

neurobiologiques sont d'une telle complexité à l'échelle de l'organisme entier que le rôle physiologique des différents systèmes est bien difficile à prévoir à partir des seules études moléculaires, physiologiques ou pharmacologiques. C'est dire que l'inactivation ou la modification ciblée des gènes codant pour les protéines étudiées est peut-être aujourd'hui une étape encore plus importante en neurobiologie que dans les autres disciplines; les exemples en sont abondants dans $\mathrm{m} / \mathrm{s}$ de ces deux dernières années. Ainsi, l'inactivation du transporteur de la dopamine, molécule essentielle de la régulation des signaux dopaminergiques, permetelle de bien mettre en évidence l'importance de la plasticité des systèmes de neurotransmission, mais aussi ses limites. Il faudra probablement attendre le résultat de telles expériences pour parfaitement comprendre le rôle respectif des deux somatostatines aujourd'hui caractérisées, et pour être assuré que le nouveau récepteur $N P Y$ identifié est bien celui qui relaie les effets stimulants de ce neuropeptide sur l'appétit.

\title{
Le transporteur neuronal de la dopamine : de la protéine à la fonction
}

La dopamine est impliquée dans le fonctionnement normal et pathologique du cerveau

Dans le système nerveux central, la transmission dopaminergique agit en réglant l'activité de certaines fonctions supérieures. Deux de ces fonctions sont particulièrement bien connues car leurs perturbations donnent lieu à des troubles du fonctionnement cérébral, conduisant à des maladies neurologiques ou psychiatriques pour lesquelles de gros efforts de recherche ont été engagés depuis plus de trente années. Ces deux fonctions sont portées par des voies nerveuses ayant leur origine dans les noyaux à plus forte densité en corps cellulaires dopaminergiques. Ainsi, la régulation de l'activité motrice est majoritairement portée par la voie dopaminergique ayant son origine dans la partie compacte de la substance noire (SNC), et se projetant dans le striatum dorsal [1]. Ce sont ces neurones dopaminergiques dont la dégénérescence est la cause principale de l'apparition des symptômes de la maladie de Parkinson, observation qui, dès les années 60 [2], avait conduit O. Hornykiewicz à proposer une thérapeutique substitutive en fournissant aux malades la L-DOPA, le précurseur direct de la dopamine, afin de suppléer au déficit de ce neurotransmetteur [3]. Quant aux neurones dopaminergiques contenus dans l'aire tegmentale ventrale (ATV), ils vont principalement se projeter dans la partie ventrale du striatum (n. accumbens et tubercules olfactifs) et le cortex frontal. Ces faisceaux méso-limbique et méso-cortical règlent les fonctions cognitives et affectives, qui sont perturbées dans des troubles psychiatriques tels que la schizophrénie et la toxicomanie. L'implication possible de la transmission dopaminergique dans la schizophrénie découle de l'observation empirique de l'efficacité des neuroleptiques dans l'amélioration des symptômes positifs de cette maladie [4]. En effet, alors que le premier neuroleptique, la chlorpromazine, est apparu dans les années 1950 [5], ce n'est que 20 ans plus tard que sa cible pharmacologique dans le cerveau a été caractérisée comme étant le récepteur dopaminergique D2 [6]. Avec l'explosion du clonage des gènes codant pour les sous-types de récepteurs dopaminergiques, il apparaît que tous les neuroleptiques ont en commun la capacité de bloquer dans le cerveau l'activité des récepteurs D2, D3 et D4, appartenant à la famille du récepteur D2 [7].

Quant à la toxicomanie, il a été démontré qu'une augmentation de l'activité des neurones dopaminergiques de la voie méso-limbique est un mécanisme commun mis en jeu lors de l'administration de drogues addictives telles que les psychostimulants (cocaïne, amphétamine), les opiacés (morphine, héroïne), l'alcool ou la nicotine [8], et que l'activation de cette voie est impliquée dans des mécanismes de récompense qui conduisent au renforcement de l'usage $[9,10]$. Cependant, les modalités d'actions de la dopamine ne sont pas encore pleinement comprises lors de ces phénomènes.

\section{La dopamine est \\ un neuromodulateur}

Si la dopamine est considérée comme un neuromodulateur, c'est 


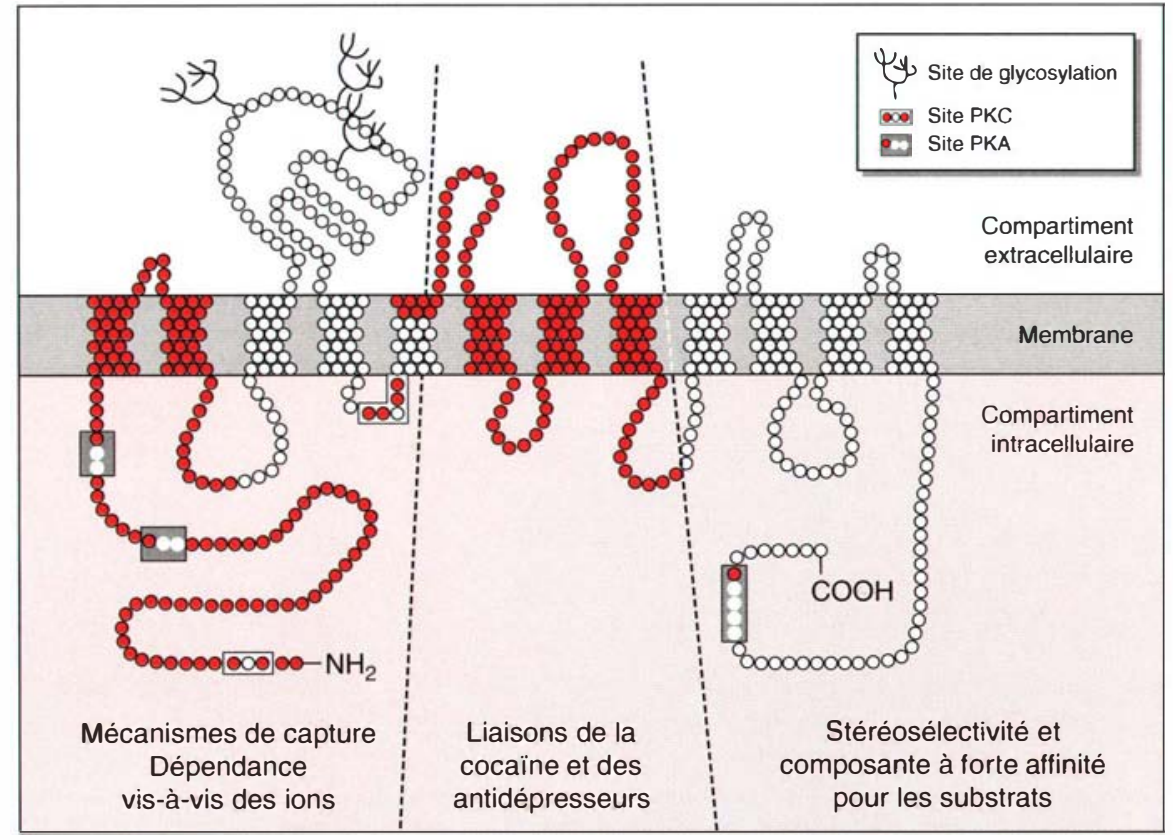

Figure 1. Représentation schématique du transporteur de la dopamine. Le transporteur dopaminergique humain comporte 620 acides aminés, représentés par des cercles. Les parties amino- $\left(\mathrm{NH}_{2}\right)$ et carboxy- $(\mathrm{COOH})$ terminales sont situés à l'intérieur de la cellule (compartiment intracellulaire), cette topologie étant la conséquence: (1) de l'absence de peptide signal et (2) de la présence potentielle de 12 domaines transmembranaires déterminés d'après le calcul de l'hydrophobicité des résidus. II existe trois sites potentiels de glycosylation, tous localisés dans la deuxième boucle extracytoplasmique. Il y a trois sites consensus de phosphorylation pour la protéine kinase $A(P K A)$, et deux pour la protéine kinase $C(P K C)$. Le transporteur de la dopamine est divisé en quatre cassettes, d'après les transporteurs chimériques qui ont été obtenus. Les différents domaines délimités par les relations "structure-activité " sont séparés par des lignes pointillées, et les fonctions qui leur sont affectées sont spécifiées.

parce qu'elle agit par l'intermédiaire de récepteurs à sept domaines membranaires couplés aux protéines G, contrairement à d'autres molécules neuroactives comme les acides aminés excitateurs (glutamate, aspartate), ou inhibiteurs (glycine, GABA), qui agissent en activant des récepteurs canaux avec pour conséquence immédiate un changement de potentiel membranaire. Alors que ces récepteurs canaux se désensibilisent très rapidement (de la $\mathrm{ms}$ à la dizaine de ms), souvent par l'action du ligand lui-même, la transmission dopaminergique (qui peut durer quelques centaines de millisecondes) se termine par une disparition physique du neurotransmetteur de l'espace synaptique, grâce à un mécanisme de recapture. Cette recapture est assurée par une protéine mem- branaire, le transporteur de la dopamine (TDA), dont l'existence est connue depuis les années 1960 [1113]. L'activité du TDA avait pu être mise en évidence, d'abord sur coupes de cerveaux, puis sur des préparations de synaptosomes, grâce à la disponibilité de dopamine radiomarquée, permettant ainsi de détecter son accumulation dans les terminaisons nerveuses [14]. Les trois amines biogènes (dopamine, noradrénaline et sérotonine) possèdent chacune un transporteur spécifique, cette spécificité étant d'ailleurs déterminée davantage par leur localisation que par la nature de leur substrat. L'énergie utilisée par ces transporteurs pour la translocation du neuromédiateur est le gradient transmembranaire de $\mathrm{Na}^{+}$. Dans les neurones, la pompe $\mathrm{Na}^{+} / \mathrm{K}^{+}$-ATPase établit la concentration intracellulaire du $\mathrm{Na}^{+}$ à $5 \mathrm{mM}$, et la concentration extracellulaire à $120 \mathrm{mM}$. Pour entrer dans la cellule en suivant la loi d'action de masse, le $\mathrm{Na}^{+}$va utiliser le transporteur, qui va tirer partie de cette énergie pour co-transporter une molécule de substrat. Ce transport étant également sensible à la concentration des ions $\mathrm{Cl}^{-}$, il est appelé transport dépendant de $\mathrm{Na}^{+} / \mathrm{Cl}^{-}$. Des études biochimiques et pharmacologiques ont montré depuis longtemps que les transporteurs de la dopamine, de la noradrénaline et de la sérotonine, et également ceux du GABA, de la glycine et de la choline appartiennent à la même famille. Il existe d'autres familles de transporteurs, comme celle des transporteurs neuronaux dépendants de $\mathrm{Na}^{+} / \mathrm{K}^{+}$(glutamate, aspartate), et celle des transporteurs vésiculaires dépendants de $\mathrm{H}^{+}$ (amines).

Les transporteurs neuronaux sont responsables (avec les enzymes de dégradation) du contrôle de la concentration synaptique du neurotransmetteur après sa libération, et donc de l'amplitude et de la durée de la transmission. Etant donné leur rôle clé à la synapse, ils sont la cible de psychotropes extrêmement actifs. Les deux exemples les plus marquants sont les psychostimulants (cocaïne, amphétamine) qui bloquent avec à peu près la même affinité les trois transporteurs aminergiques, et certains antidépresseurs, comme les antidépresseurs tricycliques [15], qui bloquent les transporteurs de la noradrénaline (NA) et de la sérotonine (5-HT) avec une meilleure affinité que le TDA. Enfin, les SSRI comme la fluoxétine (Pro$\mathrm{zac}^{\circledast}$ ) bloquent de manière spécifique le transporteur de la 5-HT.

Caractérisation moléculaire du transporteur neuronal de la dopamine

Le clonage de l'ADNc d'un transporteur du GABA après sa purification par le groupe de B. Kanner en 1990 [16], et celui d'un transporteur de la NA par des techniques d'expression par le groupe de S. Amara en 1991 [17], permit de réaliser que ces deux transporteurs dépendants de $\mathrm{Na}^{+} / \mathrm{Cl}^{-}$ 
possèdent non seulement une forte similitude structurale, mais également une identité de plus de $30 \%$ de leurs structures primaires. En utilisant des techniques d'amplification par PCR, ou de criblage de banques d'ADNc à faible stringence, tirant avantage de cette homologie, le TDA a été rapidement cloné et caractérisé chez. le rat [18-20], le bœuf [21] et l'homme [22]. Dans le même temps, à l'exception du transporteur de la choline, la plupart des transporteurs de cette famille ont vu leurs ADNc être clonés (sous-types pour le GABA, 5-HT, glycine), même certains comme ceux de la proline, de la taurine, de la bétaïne et de la créatine dont l'existence même était mal définie (pour revue, voir [23-25]).

Comme les autres membres de cette famille, le TDA est organisé en 12 domaines transmembranaires (TM) définis d'après l'analyse du profil d'hydrophobicité. Les parties amino- et carboxyterminales sont situées dans le compartiment cytoplasmique, et il existe une longue boucle extracytoplasmique entre le troisième et le quatrième domaine TM (figure 1). Le TDA humain comporte 620 acides aminés, trois sites putatifs de glycosylation sur la longue boucle extracytoplasmique, et 3 sites consensus pour la PKA et 2 sites consensus pour la PKC dans les domaines intracytoplasmiques [24]. Le TDA humain possède $98 \%$ d'identité avec celui de rat, et son profil pharmacologique pour différents inhibiteurs déterminé après expression dans des cellules eucaryotes en est extrêmement proche [22]. La seule différence observée entre le TDA recombinant et le transporteur natif étudié dans des préparations de synaptosomes de rat est la perte d'affinité d'environ dix fois (de $200 \mathrm{nM}$ à $2 \mu \mathrm{M}$ ) pour la dopamine [26], pour laquelle il n'y a pas encore d'explication à l'heure actuelle.

Le gène codant pour le TDA humain est localisé sur le chromosome 5 [22]. Étant donné l'importance de la transmission dopaminergique dans l'étiologie de certaines maladies psychiatriques, on a recherché si le gène du TDA était lié, ou associé, à certaines d'entre elles. Jusqu'à présent, rien n'a pu être mis en évidence dans le cas de la schizophrénie [27-30], mais une étude récente, qui demande confirmation, semble montrer une association entre un allèle du gène avec le syndrome d'hyperactivité (attention deficit disorder) chez. les enfants [31].

\section{Les transporteurs ont différents domaines fonctionnels}

En utilisant le fort degré d'analogie $(78 \%)$ entre les transporteurs humains pour la dopamine et la noradrénaline, il a été possible pour la première fois de construire une série de transporteurs chimériques permettant d'étudier les relations "structure-activité " entre les différents domaines et les caractéristiques fonctionnelles du transport. Bien évidemment le transporteur doit rester entier pour fonctionner correctement, et des délétions de domaines transmembranaires, ou encore de parties des boucles intermembranaires, ont pour résultats de conduire à un transporteur inactif (B. Giros, résultats non publiés). Il n'en reste pas moins que grâce à ces chimères, il est maintenant possible de diviser grossièrement le transporteur en trois domaines fonctionnels (figure 1). La partie amino-terminale, jusqu'au cinquième domaine TM, est responsable des mécanismes de recapture de la DA et de la dépendance aux ions [32, 33], alors que la partie carboxy-terminale semble plutôt impliquée dans la stéréospécificité pour les inhibiteurs, et la forte affinité pour les substrats [32, 33]. Quant à elle, la partie centrale du transporteur (TM 6 à 8) semble porter les déterminants de la liaison à forte affinité pour les antidépresseurs de la classe des tricycliques (désipramine, amitriptyline), également pour la cocaïne [33]. Cette étude des relations structure-activité pour les transporteurs des amines biogènes doit maintenant être précisée par une approche plus fine de mutagenèse dirigée, ce qui devrait à moyen terme nous apporter des informations importantes permettant un développement rationnel de nouveaux inhibiteurs de recapture plus spécifiques.

\section{Suppression du transporteur neuronal de la DA chez la souris}

Plus récemment, et dans le but de mieux connaître le rôle et la fonction du TDA, nous avons développé un modèle de souris [34] dans lequel ce transporteur a été supprimé par manipulation génétique (recombinaison homologue in vivo). Ces souris devaient, en particulier, nous permettre de mieux comprendre le rôle de la transmission dopaminergique dans le contrôle des fonctions où elle intervient.

D’un point de vue général, les animaux TI) $A^{-1-}$ sont inférieurs en taille et en poids d'environ $30 \%$ comparés aux animaux "sauvages". Ils sont également très fragiles précocement puisque $30 \%$ des souris $T I) A^{-/-}$ne survivent pas au-delà des 10 premières semaines après la naissance. Ces deux particularités pourraient être directement liées à l'hyperactivité de ces souris (voir ci-dessous), ou bien encore être une conséquence des changements de libération des hormones hypophysaires sous le contrôle de la dopamine (hormone de croissance et prolactine).

D'un point de vue biochimique et anatomique, nous avons mesuré in situ les taux d'ARNm codant pour des protéines dont on sait qu'elles sont directement impliquées dans la transmission dopaminergique (récepteurs, enzymes...), ou bien sous son contrôle direct (neuropeptides). Pour certaines protéines synthétisées au niveau des voies nigrostriée et méso-limbique, nous observons une diminution très importante de la transcription. Ainsi, pour les récepteurs Dl post-synaptiques et les D2 pré- et post-synaptiques dans le striatum, il y a une baisse de $50 \%$ du niveau de production, ce qui n'avait encore jamais été obtenu par une manipulation pharmacologique, lésionnelle ou comportementale chez l'animal d'expérience [35]. La diminution de concentration des ARNm spécifiques est accompagnée d'une réduction de la synthèse des protéines correspondantes, mesurée par un dosage direct des récepteurs (par techniques de radioliaison). Nous avons également 


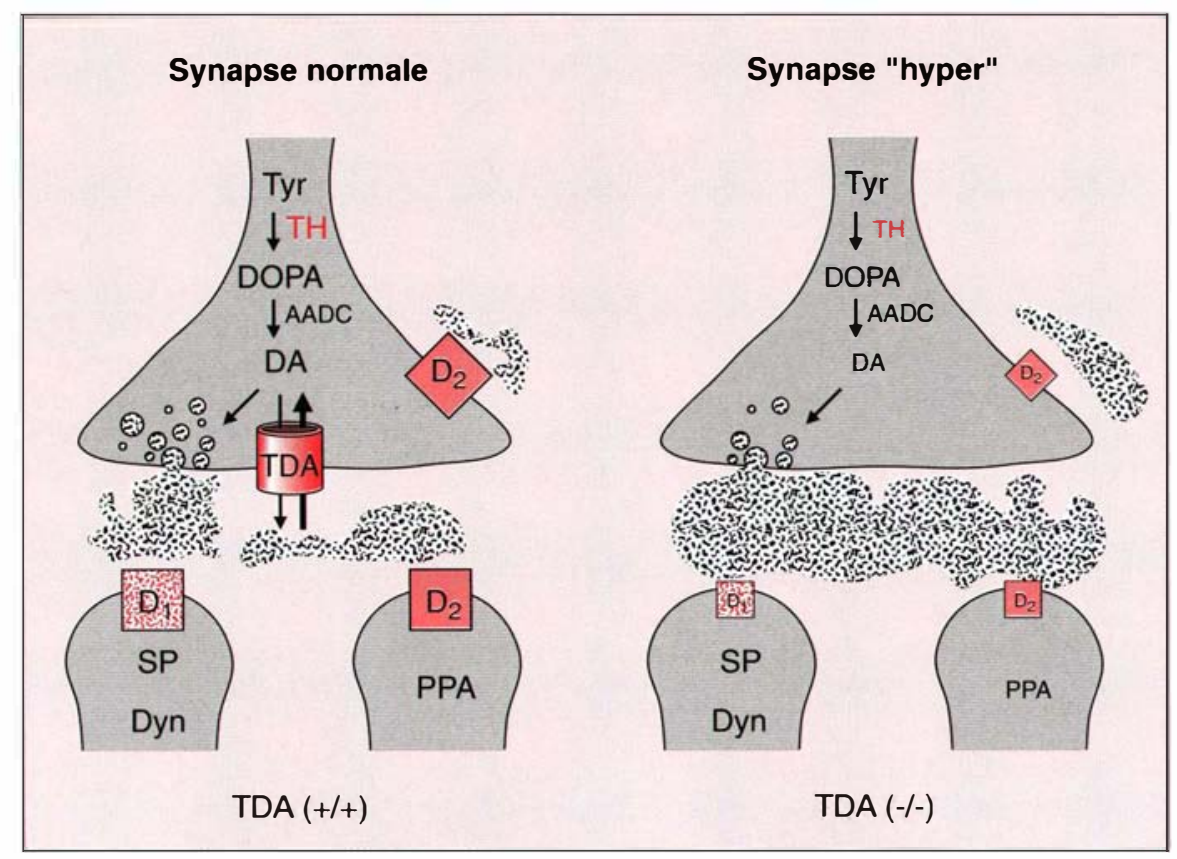

Figure 2. Conséquences moléculaires du knock-out du transporteur dopaminergique. La représentation de la synapse normale intègre les résultats obtenus en mesurant les niveaux d'expression des ARNm dans le VMB (substance noire et aire tegmentale ventrale) où sont situés les corps cellulaires dopaminergiques (élément présynaptique), et dans le striatum où sont situées les cellules dopaminoceptives (élément post-synaptique). Les niveaux d'expression de la tyrosine hydroxylase, des récepteurs D1 et D2, de la substance $P$, de la proenképhaline et de la prodynorphine ont été mesurés par hybridation in situ quantitative. Les quantités de dopamine cytoplasmique ont été déterminées par HPLC et détection électrochimique. Les quantités de dopamine dans l'espace synaptique ont été mesurées par polarographie sur coupes de tissus, après stimulation électrique. Pour les animaux DAT(-l-), les différents éléments de la synapse "hyperdopaminergique" (dont les niveaux d'expression sont modifiés) sont indiqués par un changement de la taille de leur représentation (voir texte pour les chiffres exacts). $A A D C$ : décarboxylase des acides aminés aromatiques; $D 1, D 2$ : récepteurs D1 et D2 de la dopamine; DA : dopamine; TDA : transporteur de la dopamine ; Dyn : pré-prodynorphine; PPA: pré-proenképhaline; SP: substance $P$; $\mathrm{TH}$ : tyrosine hydroxylase; tyr : tyrosine.

observé une diminution d'environ $75 \%$ des taux d'ARNm de la préproenképhaline (PPA), dont la transcription du gène est sous le contrôle inhibiteur des récepteurs D2 [35, $36]$. En revanche, alors que les gènes codant pour la pré-prosubstance $\mathrm{P}$ (SP) et la pré-prodynorphine (dyn) sont tous les deux sous le contrôle activateur des récepteurs D $1[35,37]$, nous avons observé une augmentation $(50 \%)$ des taux d'ARNm de la dyn seulement, ce qui indique probablement des mécanismes de régulation différents pour ces deux précur- tion du nombre de cellules dopaminergiques. De manière paradoxale, les quantités d'ARNm de la TH ne sont diminuées que de $10 \%$ à $20 \%$ (M. Jaber, communication personnelle), ce qui semblerait indiquer que la diminution de la protéine serait la conséquence, soit d'une baisse de la traduction, soit d'une augmentation de sa dégradation.

Des expériences de voltamétrie sur coupes de striatum nous ont permis de constater qu'après sa libération synaptique, la dopamine restait au moins 100 fois plus longtemps à la synapse chez les animaux $T D A^{-/}$que chez les témoins. En fait, elle n'en disparaît que par un mécanisme de diffusion passive. Ce phénomène est directement lié à la suppression du TDA, et constitue le phénotype majeur des souris knock-out. Sans transporteur, la dopamine va imprégner ses cibles biologiques beaucoup plus longtemps, avec les conséquences biochimiques décrites précédemment.

Considérés dans leur ensemble (figure 2), ces résultats indiquent qu'à la suite de la disparition du TDA, il y a une adaptation à l'extrême du système de neurotransmission dopaminergique. L'ensemble du système tend à compenser l'excès de dopamine synaptique par une diminution à la fois des récepteurs pré- et postsynaptiques, mais également par une diminution de plus de 10 fois de l'enzyme de synthèse. La plasticité qui est observée va bien au-delà de tout ce qui a été mis en évidence jusqu'alors; elle nous indique probablement quelles sont les limites de l'adaptabilité du système de neurotransmission dopaminergique. Il est également intéressant de noter que les animaux hétérozygotes présentent de manière significative des taux d'expression intermédiaires entre les animaux sauvages et les animaux knock-out TDA ${ }^{-1-}$, ce qui démontre une graduation et une progression dans la plasticité du système dopaminergique.

\section{Sans transporteur, les souris sont hyperactives}

L'étape suivante de l'analyse de ces animaux était l'étude comportemen- 


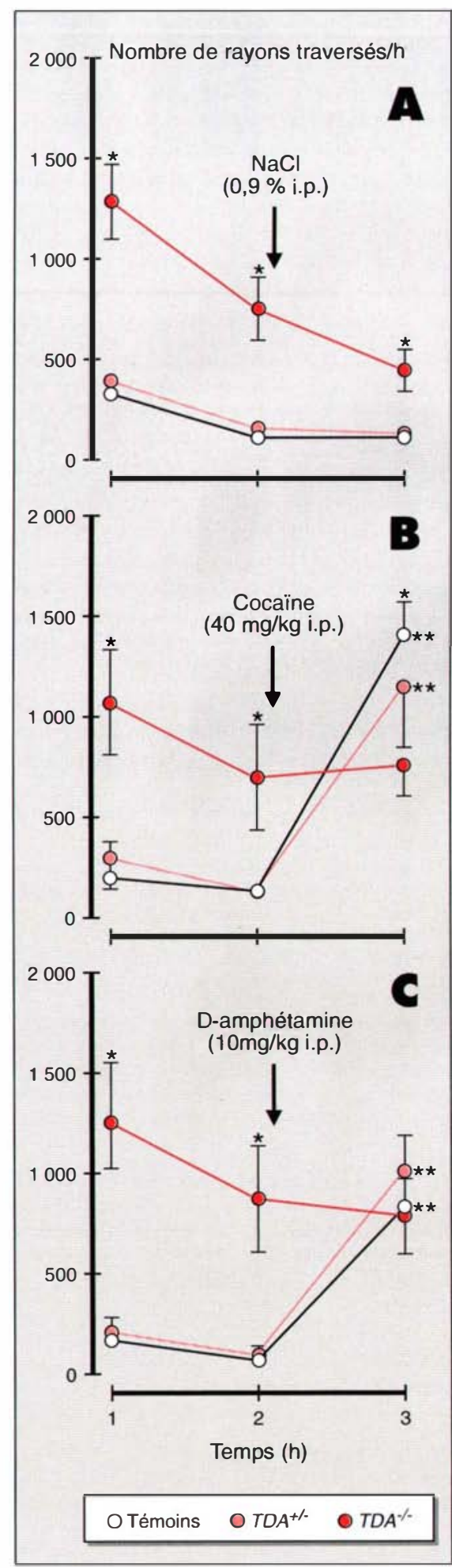

tale, la question étant de savoir si les mécanismes de compensation mis en place allaient ou non être suffisants pour masquer l'absence du transporteur de la dopamine. S'il était logique d'attendre une augmentation de la motricité chez ces animaux, l'amplitude de l'activité spontanée observée (figure 3) est extrêmement importante. Les animaux $T D A^{-/-}$présentent une activité de 5 à 6 fois supérieure à celle des animaux témoins $T D A^{+/+}$ou hétérozygotes $T I \mathrm{~A}^{+/}$, et ce aussi bien durant la période diurne que nocturne. Nous avons également pu vérifier l'absence d'effet de la cocaine et de l'amphétamine, ce qui constitue la première preuve formelle du rôle du transporteur dopaminergique dans l'effet hyperlocomoteur, à l'exclusion donc des transporteurs de la sérotonine et de la noradrénaline (qui sont pourtant également la cible de ces psychostimulants). Nous n'avons, en revanche, pas observé de stéréotypies, comme spontanément après l'injection de psychostimulants; cette observation était quelque peu surprenante pour l'amphétamine, étant donné son action stimulante sur la libération de dopamine. En fait, des expériences de voltamétrie nous ont permis de montrer que l'amphétamine utilise le transporteur «à l'envers» pour libérer la dopamine dans la synapse, et que ce mécanisme devient, bien sûr, impossible chez les souris $T I A^{-/-}$[34].

Ces animaux "hyperdopaminergiques » représentent un modèle tout à fait unique. Au-delà de ce qu'ils nous ont déjà appris sur la plasticité du système de transmission dopaminergique et sur l'effet direct des psychostimulants, deux voies de recherche tout à fait fascinantes sont à exploiter maintenant.

D'une part, il est tentant de faire un parallèle entre les symptômes pro- ductifs observés che\% les patients schizophrènes [4] et "l'hyperdopaminergie " mise en évidence chez les animaux TI) $A^{-/-}$. Des expériences préliminaires nous ont permis de montrer que l'hyperlocomotion des souris $T D A^{-/-}$pouvait être prévenue pendant plusieurs heures par l'administration d'une seule dose de halopéridol $\left(\mathrm{Haldol}^{\circledR}\right)$. Ces souris devraient donc représenter un excellent modèle pour une meilleure compréhension des mécanismes d'action des neuroleptiques, mais également offrir les moyens de comparer l'effet des neuroleptiques typiques et atypiques, c'est-à-dire des médicaments antipsychotiques qui entraînent, ou n'entraînent pas, l'apparition de symptômes extrapyramidaux (voir discussion dans [7]).

D'autre part, ces animaux «hyperdopaminergiques» offrent la possibilité, jusqu'alors inexistante, d'étudier, par une approche nouvelle et originale, le rôle de la dopamine dans une fonction supérieure intégrée qui est l'élaboration des circuits de motivations, et plus exactement les mécanismes de récompense et de renforcement positif $[9,10]$. Si, en effet, il est connu depuis longtemps que le mécanisme commun qui suit l'administration de drogues comme la cocaïne, la morphine, l'éthanol, ou encore la nicotine, est la libération de dopamine dans l'écorce du noyau accumbens [8], en revanche, les tenants et les aboutissants de ce phénomène sont encore mal définis. Le modèle des souris $T V A^{-1-}$ devrait permettre, par comparaison avec des souris normales, de mieux comprendre le rôle précis de la dopamine dans les mécanismes moléculaires et cellulaires qui sous-tendent la dépendance aux drogues, et donc de proposer à terme des stratégies thérapeutiques alternatives 


\section{Bruno Giros}

Directeur de recherche au Cnrs, Inserm U. 288, 91, boulevard de l'Hôpital, 75013 Paris, France.

\section{TIRÉS À PART}

B. Ciros.

\section{Remerciements}

le voudrais particulièrement remercier le support constant et enthousiaste du Pr M.G. Caron (Duke University, NC, USA), sans lequel ces résultats n'auraient pas été obtenus.

\section{RÉFÉRENCES}

1. Anden NE, Dahlstrom A, Fuxe K, Larsson K. Further evidence for the presence of nigro-neostriatal dopamine neurons in the rat. Am J Anat $1965 ; 116$ : 329-33.

2. Ehringer $\mathrm{H}$, Hornykiewicz $\mathrm{O}$. Verteilung von Noradrenalin und Dopamin (3hydroxytyramin) im gehirn des Menschen und ihr verhalten bei erkrankungen des extrapyramidalen Systems. Klin Wochenschr 1960;38: 1236-9.

3. Birkmayer W, Hornykiewicz O. Der LDioxyphenylalanin (DOPA). Effekt bei der Parkinson-akinese. Wien Klin Wochenschr $1961 ; 73$ : 787-8.

4. Crow TJ. The biology of schizophrenia. Experientia 1982; 38: 1275-82.

5. Delay J, Deniker P, Harl JM. Utilisation en thérapeutique psychiatrique d'une phénotiazine d'action centrale élective (4560RP). Ann Med Psychol 1952; 110: 112-7.

6. Seeman P, Chau-Wong M, Tedesco J, Wong $K$. Brain receptors for antipsychotic drugs and dopamine: direct binding assay. Proc Natl Acad Sci USA 1975; 72: 4376-80.

7. Sokoloff P, Martres MP, Schwartz JC. La famille des récepteurs de la dopamine. médecine/sciences $1993 ; 9$ : 12-20.

8. Di Chiara G, Imperato A. Drugs abused by humans preferentially increase synaptic dopamine concentrations in the mesolimbic system of freely moving rats. Proc Natl Acad Sci USA 1988; 85: 5274-8.

9. Stinus L, Cador M, Le Moal M. Interaction between endogenous opioids and dopamine within the nucleus accumbens. Ann NY Acad Sci 1992; 654: 254-73.

10. Wise RA, Bozarth MA. A psychomotor stimulant theory of addiction. Psychol Rev 1987; 94: 469-92.
11. Iversen LL. Role of transmitter uptake mechanisms in synaptic neurotransmissions. Br J Pharmacol 1971 ; 41 : 571-91.

12. Axelrod J. The metabolism, storage and release of catecholamines. Recent Prog Hormone Res 1965; 21 : 597-619.

13. Glowinski J, Iversen LL. Regional studies of catecholamines in the rat brain III. Subcellular distribution of endogenous and exogenous catecholamines in various brain regions. Biochem Pharmacol 1966; 15: 977-87.

14. Coyle JT, Snyder SH. Catecholamine uptake by synaptosomes in homogenates of rat brain: stereospecificity in different areas. JPharmacol Exp Ther 1969; 170: 221-31.

15. Glowinski J, Axelrod J. Inhibition of uptake of tritiated noradrenaline in the intact rat brain by imipramine and structurally related compounds. Nature 1964; 204 : 1318-9.

16. Guastella I, Nelson N, Nelson H, Czyzyk, Keynan S, Miedel MC, Davidson N, Lester HA, Kanner BI. Cloning and expression of a rat brain GABA transporter. Science 1990; 249: $1303-6$

17. Pacholczyk T, Blakely RD, Amara SG. Expression cloning of an antidepressantsensitive human noradrenaline transporter. Nature $1990 ; 350: 350-3$.

18. Giros B, El Mestikawy S, Bertrand L, Caron MG. Cloning and functional characterization of a cocaine-sensitive dopamine transporter. FEBS Letters 1991 ; 295 : 149-54.

19. Shimada S, Kitayama S, Lin CH, Patel A, Nanthakumar E, Gregor P, Kuhar M, Uhl G. Cloning and expression of a cocaine-sensitive dopamine transporter complementary DNA. Science 1991 ; 254: 576-8.

20. Kilty JE, Lorang D, Amara SG. Cloning and expression of a cocaine - sensitive rat dopamine transporter. Science 1991; 254: 578-9.

21. Usdin TB, Mezey E, Chen C, Brownstein $\mathrm{J}$, Hoffman BJ. Cloning of a cocaine sensitive bovine dopamine transporter. Proc Natl Acad Sci USA 1991 ; 88: 11168-71.

22. Giros B, Mestikawy SE, Godinot N Zheng K, Han H, Yang-Feng T, Caron MG Cloning, pharmacological characterization and chromosomal assignment of the human dopamine transporter. Mol Pharmacol 1992 42: $383-90$

23. Amara SG, Kuhar MJ. Neurotransmitter transporters: recent progress. Annual Rev Neurosci 1993; 16: 73-93.

24. Giros B, Caron MG. Molecular characterization of the dopamine transporter. Trends Pharmacol Sci 1993 ; 14 : 43-9.

25. Uhl GR, Hartig PR. Transporter explosion: update on uptake. Trends Pharmacol Sci $1992 ; 13$ : 421-5.
26. Pifl C, Giros B, Caron MG. Dopamine transporter expression confers cytotoxicity to low doses of the parkinsonism-inducing neurotoxin 1-methyl-4-phenylpyridinium. J Neurosci 1993; 13: 4246-53.

27. Persico AM, Wang ZW, Black DW Andreasen NC, Uhl GR, Crowe RR. Exclusion of close linkage of the dopamine transporter gene with schizophrenia spectrum disorders. Am J Psychiatr 1995; 152: 134-6.

28. Byerley W, Hoff M, Holik J, Caron MG, Giros B. VNTR polymorphism for the human dopamine transporter gene (DAT1). Hum Mol Genet 1993; 2: 335.

29. Byerley W, Coon H, Hoff M, Holik J, Waldo M, Freedman R, Caron MG, Giros B. Human dopamine transporter gene not linked to schizophrenia in multigenerational pedigrees. Hum Hered 1993; 43: 319-22.

30. Li T, Yang L, Wiese C, Xu CT, Zeng Z, Giros B, Caron MG, Moises HW, Liu X. No association between alleles or genotypes at the dopamine transporter gene and schizophrenia. Psychiatr Res 1994; 52 : 17-23.

31. Cook E Jr, Stein MA, Krasowski MD, Cox NJ, Olkon DM, Kieffer JE, Leventhal BL. Association of attention-deficit disorder and the dopamine transporter gene. Am J Hum Genet 1995; 56: 993-8.

32. Buck KJ, Amara SG. Chimeric dopamine-norepinephrine transporters delineate structural domains influencing selectivity for catecholamines and 1-methyl-4-phenylpyridinium. Proc Natl Acad Sci USA 1994; 91 : 125848

33. Giros B, Wang YM, Suter S, McLeskey SB, Pifl C, Caron MG. Delineation of discrete domains for substrate, cocaine, and tricyclic antidepressant interactions using chimeric dopamine-norepinephrine transporters. J Biol Chem 1994; 269: 15985-8.

34. Giros B, Jaber M, Jones SR, Wightman RM, Caron. MG. Hyperlocomotion and indifference to cocaine and amphetamine in mice lacking the dopamine transporter. Nature 1996; 379: 606-12.

35. Gerfen CR, Engber TM, Mahan LC, Susel Z, Chase TN, Monsma F Jr, Sibley DR. D1 and D2 dopamine receptor-regulated gene expression of striatonigral and striatopallidal neurons. Science 1990; 250: 1429-32.

36. Le Moine $\mathrm{C}$, Normand $\mathrm{E}$, Guitteny AF, Fouque B, Teoule R, Bloch B. Dopamine receptor gene expression by enkephalin neurons in rat forebrain. Proc Natl Acad Sci USA $1990 ; 87: 230-4$.

37. Le Moine C, Normand E, Bloch B. Phenotypical characterization of the rat striatal neurons expressing the D1 dopamine receptor gene. Proc Natl Acad Sci USA 1991; 88: 4205-9. 